\title{
Nursing Treatments and Collaboration of Postpartum Hemorrhage Patients as Efforts to Prevention of Maternal Mortality: A Literature Review
}

\author{
1.2. Diyan Indriyani, ${ }^{3 .}$ Moses Glorino Rumambo Pandin, Dr. M.Si., M.Phil., M.Psi., Psikolog \\ ${ }^{1 .}$ Student of Doctoral in Nursing, Faculty of Nursing, Airlangga University \\ ${ }^{2 .}$ Faculty of Health Science University of Muhammadiyah Jember \\ ${ }^{3 .}$ Departement of English Literature, Faculty of Humanities, Airlangga University
}

Email : diyan.indriyani-2020@fkp.unair.ac.id

\begin{abstract}
Postpartum hemorrhage is a complication during the puerperium that requires serious management, because it risks on threatening the mother's death. Currently, postpartum hemorrhage is still the main cause of maternal morbidity and mortality in the world. There is a need for a fast, precise and comprehensive handling effort involving all elements, such as the entire health team including doctorsnurses and families. The form of nursing intervention in postpartum hemorrhage patients can be in the form of nursing treatment or collaboration. The purpose of this review literature is to identify nursing treatment and nursing collaboration in postpartum hemorrhage patients as an effort to prevent maternal death. The writing method uses literature reviews by summarizing 10 articles publicated in 2019, 2020 and 2021 in 4 search data bases including Sciencedirect, Scopus, Proquest and Thompson. The review guidelines used are based on Prisma and the Joanna Briggs Institutute. The level of eligibility is identified through the title, abstract, research methodology and full text. The results of the reviews found are presented in a narrative form. The results of the review study found that there were 10 articles on postpartum hemorrhage management measures both in the effort of snoring treatment and collaboration including: optimizing the competence of service providers based on E_Learning, foot reflexology since the 4th stage of labor, $800 \mathrm{mcg}$ sublingual Misoprostol, the use of the Digital Postpartum Hemorrhage Management Device ( DPHMD), bilateral-contralateral cervical clamps, suction tube uterine tampons, bilateral hypogastric artery (BHAL), evaluation of Shock Index, Carbetocin drugs and administration of Tranexamic acid 1 gram IV within 10 minutes of vaginal delivery. The conclusion of this review literature is that nursing treatment and nursing collaboration are proven to have a positive impact as an effort to prevent death in postpartum hemorrhage patients.
\end{abstract}

Keywords: Nursing Treatments; Nursing Collaboration; Postpartum Hemorrhage; Maternal Mortality

\section{INTRODUCTION}

Childbirth is a phase where a special attention from medical staff and family are important in order to identify postpartum compilation early, in doing so, the chance of maternal morbidity and mortality during childbirth can be reduced. The death rate of expectant mother is still taking a top priority and vigilance that are important for the health of maternity (1). One of the highest reasons of maternity death is postpartum compilation (postpartum hemorrhage) (2).

Postpartum Hemorrhage (PPH) is the leading cause of maternal morbidity and mortality worldwide (3). There are many factors that can cause Postpartum Hemorrhage, they are the process of the delivery, uterine atony, maternal comorbidities (4), the age of expectant mother which is 35 or above, childbirth complication, and whether expectant mother have a history of 
Postpartum Hemorrhage before, all those factors mention before act as an indicator to predict the Primary Postpartum Hemorrhage (5). Considering that Primary Postpartum Hemorrhage is the leading cause of maternal morbidity and mortality, it is important to improve on the skill and quality of medical practitioners in order to identify and give a proper response for Postpartum Hemorrhage (6).

There are plentiful efforts that can be made to prevent the death of expectant mother due to Postpartum Hemorrhage. Starts from intervention in the form of observation, medical education, and through independent action or collaboration. Individual risk factors are poor predictor of Postpartum Hemorrhage (PPH), because of that special attention in nursing practices on patient management is also needed as this factor can affect maternal morbidity and mortality (7). The management of conservative PPH generally include resuscitation, blood transfusion, administration of uterotonic drug such as oxytocin and prostaglandins, uterine compression and the usage of intrauterine tamponade (8). During bleeding, it is necessary not only to maintain circulating blood volume and tissue oxygenation, but also to support hemostasis (9).

Considering that there are so many efforts and procedures needed to be made by health care providers to prevent the incidence of death in patients who suffer from Postpartum Hemorrhage, it is important to do a literature-based research based on research result. The aim of the research itself is to gain a main conclusion on how to counter and handle Postpartum Hemorrhage.

\section{METHOD}

Through this article, the method used in writing this literature is to make a comprehensive summary of 10 articles that have been reviewed with the following methods:

\section{Study Protocol}

This protocol is a quality identifier technique with the usage of PRISMA table and Institute of Joanna Briggs guideline based on the theme that are being researched.

\section{Article Search Strategy}

The article search strategy used searches on 4 electronic databases such as Scopus, Science Direct, Thomson and ProQuest Journal. The keywords used are based on the chosen theme, namely "intervention-implementation-therapy-protocol" and "postpartum hemorrhage".

\section{Inclusion Criteria}

In connection with the article inclusion criteria that have been defined are interventionpostpartum hemorrhage with the provisions in the form of: journal topics about InterventionPostpartum Hemorrhage published in 2019, 2020 and 2021, using English full text access, quantitative and qualitative research designs with populations and samples as well as variable related to "intervention-implementation-therapy-protocol" and "postpartum hemorrhage". As a guide in reviewing this journal, use the Joanna Briggs Institute (JBI) for articles on Qualitative designs and the PRISMA guide as a review guide for articles that have Quantitative designs with eligibility study from title, abstract, methodology, results and full text. 


\section{RESULTS}

The search results obtained 45 articles that match the keywords, then checked for duplication and found the same 14 articles so that the remaining 36 articles. Furthermore, screening was carried out based on title $(n=36)$, abstract $(n=25)$ and full text $(n=10)$. The identification based on the eligibility of the inclusion and exclusion criteria was obtained as many as 10 articles with the theme of postpartum intervention and quantitative and qualitative research designs on 4 databases of electronic search, namely Scopus, Science direct, Thompson and Proquest. The results obtained are presented in a narrative manner as follows:

1. The journal title is "A Cluster-Randomized, Non-Inferiority Trial Comparing Use Of Misoprostol For Universal Prophylaxis Vs. Secondary Prevention Of Postpartum Hemorrhage Among Community Level Births In Egypt". The background to this journal is community-based research, which previously showed that secondary prevention of postpartum hemorrhage with misoprostol was only given to women with above average blood loss, resulting in a similar clinical condition compared to routine administration of misoprostol for prevention of postpartum hemorrhage. Considering the difficulty of routinely measuring blood loss for all deliveries, a more operational model of secondary prevention is needed. The method was carried out in parallel trials with randomized groups starting in November 2015 to January 2016 on mothers who gave birth assisted by midwifery nurses in the villages of Kafr el Dawar and Damanhour, El Beheira Province, Egypt. Criteria for respondents who were taken were mothers who performed ANC in the 3rd trimester, plan to give birth at home or a public health center assisted by a midwifery nurse, willing to do $\mathrm{Hb}$ checks before and after delivery, willing to conduct follow-up interviews, not allergic to Misoprostol or Prostaglandins. Two management approaches for postpartum hemorrhage were performed and compared: 1) oral misoprostol $600 \mathrm{mcg}$ was given to all women after delivery (i.e. primary prevention, current standard of care); 2) Sublingual misoprostol $800 \mathrm{mcg}$ should only be given to women with $350-500 \mathrm{ml}$ postpartum blood loss (i.e. .secondary prevention). The main outcome was the mean change in hemoglobin before and after delivery. Secondary outcomes included hemoglobin $\geq 2 \mathrm{~g} / \mathrm{dL}$ and other postpartum hemorrhage intervention. The results showed that Misoprostol was administered postpartum to $100 \%(1555 / 1555)$ and $10.7 \%$ $(117 / 1099)$ of women in the primary and secondary prevention groups. The mean reduction in hemoglobin before and after delivery was 0.37 (SD: 0.91) and 0.45 (SD: $0.76)$ among women in the primary and secondary prevention groups, respectively (differences adjusted for grouping $=0.01$, one-sided 95\% CI: $<0.27, \mathrm{p}=0.535$ ). There were no statistically significant differences in secondary outcomes, including decreased hemoglobin $\geq 2 \mathrm{~g} / \mathrm{dL}$, diagnosis of $\mathrm{PPH}$, transfer to a higher grade, or other interventions. The limitations of this journal are unequal enrollment of mothers in public health services between primary and secondary prevention groups, randomization for this study was carried out by the Primary Health Unit, which could lead to bias if populations differ greatly by Primary Health Unit, however, comparison of baseline characteristics with the primary and secondary prevention groups did not show any important differences that would affect the study results, and the researcher used the appropriate 
statistical method (i.e. the generalized approximate equation) to adjust the investigator's analysis into the grouping. This study was corroborated by the systematic measurement of hemoglobin before and after delivery, which made it possible to clearly compare the physiological effects of postpartum blood loss between respondents in the two groups. (10)

2. Title "Digital Postpartum Hemorrhage Management Device (DPHMD. Background to this journal Background: Primary Postpartum Hemorrhage / PPH) is an obstetric emergency caused by excessive blood loss that most often occurs after the placenta is born causing volume depletion, hypovolemic shock, anemia, and is the leading cause of maternal mortality worldwide. With 470 deaths per 100,000 live births, the maternal mortality ratio in Ethiopia is one of the highest in the world. An estimated $94 \%$ of births occur at home in Ethiopia and $10 \%$ of maternal deaths are due to PPH. Currently, doctors use visual estimation to calculate blood loss and deliver fluids during delivery. This traditional method is subjective and generally inaccurate. The method used is the proposed solution using an integrated postpartum blood loss measurement system by giving fluids and methods of monitoring vital signs. A collection and measurement system that collects postpartum blood loss and measures the amount of blood lost is a management system for monitoring mothers' heart rate and blood pressure on a continuous basis. This vital sign value is integrated with the measured blood loss to estimate the amount of IV fluid needed to be given to the patient. The results show that the prototype built has undergone various tests and iterations. The proposed device is tested for accuracy, cost-effectiveness and ease of use. The results show that the accuracy rate has reached $91.28 \%$, and the prototype was built for less than 210 USD. The proposed prototype design allows doctors to accurately estimate blood loss and administer fluid therapy. This helps reduce maternal mortality that may occur due to postpartum hemorrhage (11)

3. Title: "The New Clinical Application Of Bilateral-contralateral Cervix Clamp In Postpartum Hemorrhage: A Retrospective Cohort Study". The background underlying this study is that postpartum hemorrhage (PPH) has always been the leading cause of maternal mortality globally, almost resulting in $34 \%$ of 275,000 maternal deaths worldwide in 2015 , and as high as $17.62 \%$ of maternal deaths in China nationally. The most recent recommendation from the World Health Organization (WHO) to prevent postpartum hemorrhage is $10 \mathrm{IU}$ of oxytocin for all births, and uterotonics such as carbetosine, ergometrine and misoprostol can help effectively contract the uterus. However, oxytocin desensitization may decrease effectiveness, and uterotonics also have drug contraindications and side effects such as water poisoning, nausea, vomiting, and increased blood pressure, so it is important to seek other conservative treatments such as a bilateral contralateral cervical brace. To assess the efficacy and safety of first-time bilateral-contralateral cervical clamps applied to postpartum hemorrhage caused by lower segment uterine protrusion. The method used was a total of 69 pregnant women with postpartum hemorrhage from 1190 deliveries due to uterine atony. Among them, 22 women were excluded because $9 \mathrm{PPH}$ occurred during cesarean section, 8 refused to participate and others had abnormal coagulation or severe cervical laceration / erosion. Finally 27 cases of PPH during vaginal delivery and 20 after vaginal / cesarean delivery 
were included. According to the principle of informed consent, it was stated that 22 women agreed to receive cervical brace and 25 participants in the control group did not want to use uterotonics. This bilateral-contralateral cervical brace will be performed when the first uterotonic failure or women with uterotonic contraindications. Results found that mean blood loss in the bilateral-contralateral cervical brace group was significantly less during vaginal delivery $(656.2 \pm 72.79 \mathrm{~g}$ vs $811.8 \pm 86.07 \mathrm{~g}, \mathrm{p}=0.001)$ or after cesarean delivery. $(42.8 \pm 6.60 \mathrm{~g}$ vs $126.3 \pm 86.97 \mathrm{~g}, \mathrm{p}=0.007)$, and the incidence of uterotonic repeated use $(81.8 \%$ vs $36,18.2 \%$ vs $64 \%, p=0.001)$ or side effects $(18.2 \%$ vs $48.0 \%, \mathrm{p}=0.031)$ appeared to be less than the control group, but there was no statistical difference regarding hospitalization $(4.1 \pm 1.57$ days vs $3.8 \pm 1)$. , 61 days, $\mathrm{p}=0.535)$, hemoglobin $(119 \pm 4.10 \mathrm{~g}$ vs $121.4 \pm 4.19 \mathrm{~g}, \mathrm{p}=0.058)$, blood transfusion $(9.1 \%$ vs $12 \%, \mathrm{p}=0.746)$, surgical procedures $(4.5 \%$ vs $4.0 \%, \mathrm{p}=0.93)$, there were also no brace complications. So that it can be concluded that bilateralcontralateral cervical brace is effective and safe, this new technique can be a complementary therapy for postpartum hemorrhage (12).

4. Title "Novel Suction Tube Uterine Tamponade For Treating Intractable Postpartum Haemorrhage: Description Of Technique And Report Of Three Cases". Background of this study Uterine balloon tamponade (UBT) is widely used to treat postpartum hemorrhage $(\mathrm{PPH})$. Improvised UBT randomized trial with a low resource setting found more risks than benefits. Uterine suction tamponade (UST) is more attuned to the physiological mechanisms of uterine hemostasis (contraction), but specially designed devices may be out of reach or unavailable when needed. So the UST technique using an inexpensive Levin suction catheter is explained. The method used The wide hole Levin suction pipe (FG24 to FG36) is connected by the inlet pipe to an adjustable electronic suction pump. The patient's cervix is visualized with a sim speculum. The vagina and cervix are cleaned with an antiseptic solution. Reported suction pressures for specially designed devices range from $70 \mathrm{mmHg}$ to $700 \mathrm{mmHg}$. Investigators have used pressures between 100 and $200 \mathrm{mmHg}$ for suction tube uterine tamponade (STUT). If the bleeding is controlled by suction, STUT is continued for 1 hour. Then the suction is disconnected from the tube, which is left in place. If there is no significant bleeding after 20 minutes, the suction is reattached to check that no blood has collected in the uterus. If not, the suction is disconnected and the tube is removed. If bleeding continues, the suction is reconnected and rechecked every hour for up to 4 hours. This trial was conducted in 3 cases of patients with postpartum hemorrhage. The results demonstrated the use of the STUT technique described as a last resort in three cases of life-threatening postpartum hemorrhage, resulting in immediate cessation of bleeding. However, there is still a need for randomized trials to ensure their effectiveness (13)

5. Title "The Effect Of Foot Reflexology In The Fourth Stage Of Labor On Postpartum Hemorrhage And After Pain: Study Protocol For A Randomized Controlled Trial. Based on the research background, Postpartum hemorrhage is a maternal hemorrhage that most often occurs in $1 \%-5 \%$ of deliveries, causing around 140,000 deaths per year. Pain after childbirth is also one of the most common postpartum symptoms complained of about $70 \%$ of women with vaginal deliveries. Because chemical drugs have side effects, researchers have a special desire to use traditional and complementary therapies. Foot 
reflexology is one method that we want to try out. A randomized clinical trial will now be conducted to assess the effectiveness of foot reflexology on bleeding and after pain after vaginal delivery. Methods A randomized control-group trial was conducted on women giving birth in Iran. After obtaining informed consent from eligible women, subjects were randomly divided into intervention and control groups with four permutation blocks. The sample size was 40 per group. After the placenta was separated, the intervention group was given general massage for four minutes on each leg, followed by two minutes of specific reflexology massage to the uterus, pituitary point and solar plexus using rotational pressure. The control group will also be given a general massage of four minutes on each foot, followed by six minutes of rotational pressure on the point on the outer edge of the heel. Ten minutes are required for each leg, and this procedure must be repeated two hours after delivery. After that pain was measured using the Visual Analogue Scale (VAS) and post-partum bleeding by weighing the pads every one to four hours. Data were analyzed using SPSS. The results show that reflexology has a positive effect on uterine contractions and reduces pain by increasing the secretion of endorphins and enkephalins. This study aims to determine the effect of foot reflexology on the fourth stage of labor, reflexology can control postpartum hemorrhage and post partum pain. In addition, it has also been proven to be effective and safe, so that the routine use of this technique for women at the fourth stage of labor will be supported (14)

6. Title "Implementing E-Learning to Enhance the Management of Postpartum Hemorrhage". The background of this research is that the World Health Organization has stated that maternal mortality is a global public health problem. According to WHO (2019) between 2000 and 2017, MMR increased by $2.6 \%$ to 19 deaths per 100,000 births in the United States, one of which was due to postpartum hemorrhage. Remedial efforts focused on strengthening clinical response and management of maternal emergencies can help prevent maternal morbidity and mortality. Educational interventions are a common strategy for addressing inequalities, but not all education is clinically effective. The aim was to determine whether perinatal outcomes associated with postpartum hemorrhage could be improved by combining existing strategies with the use of online assessmentbased e-learning platforms. The method used was the "Institute for Healthcare Improvement Model" design as an activity for performance improvement projects. The evaluation of results was further supported by the Kirkpatrick Model. Reports of increasing maternal morbidity and mortality in the United States are prompting action in multisite health systems. The maternity nursing care team is committed to proactively supporting excellence in practice through enhancing continuing education. Participants are maternity service providers and nurses who practice in the hospital to complete the training. Interventions were measured online, based on learning module assessments for maternity emergencies combined with instructor-led courses, simulations, and Team Strategies to Enhance Performance and Patient Safety (TeamSTEPPS) training in early 2017. In addition, the postpartum hemorrhage safety package already implemented. As outcome measures included bleeding rates, massive transfusions, and admission to the intensive care unit for women being treated for delivery. Outcome measures were tracked using a retrospective chart review with a baseline period of 1 October 2016 to 31 March 2017, and a performance period of 1 April 2017 to 31 March 2018. The results measured were observations of improvement in perinatal outcomes. The mean bleeding rate 
decreased by $3 \%$ (from $56.4 / 1,000$ to $54.7 / 1,000$ ). The average massive transfusion rate decreased by $35 \%$ (from $2.3 / 1,000$ to $1.5 / 1,000$ ). Likewise, the median rate of maternal intensive care unit admissions decreased by $77 \%$ (from $3.1 / 1,000$ to $0.7 /$ $1,000)$. There was a decline supported by the admission of intensive care units which was zero for 6 out of the last 7 months $(n=4,422)$ Conclusion: there is an advantage in the management of postpartum hemorrhage supported through multiple approaches that include the use of an online e-learning platform for maternity emergencies) (15).

7. The title "Hypogastric artery ligation in postpartum haemorrhage: a ten-year experience at a tertiary care center". The background of this study investigated patients who underwent bilateral hypogastric artery ligation (BHAL) due to postpartum hemorrhage (PPH). Patients who underwent BHAL due to PPH after conservative treatment were included in this study. Placenta disorders are referred to as placenta accreta. The method used was patients who gave birth at the Gynecology and Obstetrics Clinic of the Dicle University School of Medicine and who underwent BHAL due to PPH after conservative treatment between January 2007 and December 2017, and patients who were referred to our clinic from an external center for PPH management, were included in this study . Patients who gave birth before 20 weeks of gestation and those who underwent BHAL for gynecological reasons were excluded from the study. The hypogastric artery is tied at a point $4 \mathrm{~cm}$ distal to the junction of the common iliac artery. In patients with uterine rupture, it is determined that repair of the rupture is performed first. It was found that uterine artery ligation was performed mainly in patients with uterine atony. First, active suturing of the placenta was performed in cases of placenta accreta. In cases where bleeding continues, BHAL is performed. Statistical analysis was performed using the SPSS 21.0 program and statistics were presented descriptively. Results showed a total of 130 BHAL procedures were performed in the study hospital due to PPH. Of these, 39 $(30 \%)$ were referred to the hospital. The BHAL requirement rate is 62 out of 10,000 births. Among 130 patients, the most frequent indication for BHAL was placenta accreta (58.5\%). Haematological parameters were worse among referral patients. Four of the exitus patients $(80 \%)$ were referral patients. The mortality rate for referral patients was $10.25 \%$, while this figure was only $1.01 \%$ for patients who gave birth in the hospital. $\mathrm{PPH}$ is a life-threatening condition that requires immediate medical attention. BHAL, with the impact of maintaining reproductive function (fertility), is a good option that can be applied to all PPH patients. BHAL not only protects patients from reproductive organ fertility, but also gives them a higher chance of survival (16).

8. Title "Importance of Shock Index in the Evaluation of Postpartum Hemorrhage Cases That Necessitate Blood Transfusion". Based on the background, Postpartum hemorrhage $(\mathrm{PPH})$ is the main cause of maternal morbidity and one of the top five causes of maternal death in high and low income countries. Therefore, various obstetric early warning systems (OEWS) were established to facilitate the timely recognition and treatment of maternal complications in the peripartum period. This study aims to determine the cutoff value of the shock index (SI) to predict the need for transfusion and side effects combined in cases of postpartum hemorrhage (PPH). Methods of study on one hundred and thirty $\mathrm{PPH}$ cases (study group) requiring retrospective blood transfusion were 
compared with a frequency-matched control group $(\mathrm{n}=130)$. Receiver operating characteristic (ROC) curve and decision tree [Classification \& Regression Tree (C\&RT) and Chi-square Automatic Interaction Detector (CHAID)] were used to identify the cutoff value for SI. The results obtained the cutoff values for postdelivery, peak and delta SI values for prediction of PPH requiring transfusion were 0.9125 (sensitivity 0.815 , specificity 0.923 ), 0.9145 (sensitivity 0.892 , specificity 0.823 ) and 0.195 (sensitivity 0.823 , specificity 0.885 ), 0.737 specificity) and 0.487 (sensitivity 0.710 , specificity 0.758). Delta SI was superior to postdelivery and peak SI in the prediction of PPH requiring transfusion. Peak SI was superior to postdelivery SI and delta SI in the prediction of combined adverse outcomes. In conclusion, increases in postdelivery, peak, and delta SI scores were associated with poor outcomes for PPH. SI appears to be a practical and effective method for the objective assessment of postpartum hemorrhage (17).

9. Title "Carbetocin Versus Oxytocin for Prevention Of Postpartum Hemorrhage In Hypertensive Women Undergoing Elective Cesarean Section". The background of bleeding is a major contributor to maternal mortality, especially in developing countries, accounting for more than $30 \%$ of the direct cause. Many studies have evaluated the impact of arsenal on postpartum hemorrhage (PPH), but those evaluating the effectiveness of carbetosine in hypertensive patients are rare. The steps made for the management of PPH are very clear considering uterotonic agents as an important item in the care package at the third stage of delivery. Carbetosine has advantages over oxytocin, and has minimal effect on the hemodynamics of the patient. Therefore, the current study was undertaken to evaluate the effectiveness of carbetosine versus oxytocin for prevention of postpartum hemorrhage in hypertensive women undergoing elective cesarean section. The method is to design a randomized clinical trial, in place of the Department of Obstetrics and Gynecology at the Suez Canal University Hospital. Patients: 160 hypertensive pregnant women underwent SC. Intervention: Patients were randomized to receive $10 \mathrm{IU}$ of oxytocin or $100 \mu \mathrm{g}$ carbetosine. Primary outcomes included estimates of blood loss, blood transfusion, hemoglobin (HB), and hematocrit changes before and after delivery and use of additional uterotonics. The results showed that postoperative HB was not different from preoperative HB in the carbetosine group $(11.8 \pm 1.2$ vs $11.2 \pm 1.2 \mathrm{~g} / \mathrm{dL})$. Meanwhile, it decreased significantly in the oxytocin group $(12.1 \pm 3.8$ vs $10.4 \pm 1.1 \mathrm{~g} / \mathrm{dL}, \mathrm{p}<0.001)$. Blood loss was significantly more among the oxytocin group $(679.5 \pm 200.25$ vs $424.75 \pm 182.59 \mathrm{ml})$ in the carbetosine group ( $\mathrm{p}<0.001$ ). Nausea, vomiting, and sweating were reported to be significantly more in the oxytocin group patients. So it can be concluded that Carbetosine is more effective than oxytocin in reducing intraoperative and postoperative blood loss (18).

10. Title "Prophylactic Use Of Tranexamic Acid After Vaginal Delivery Reduces The Risk Of Primary Postpartum Hemorrhage". The background is that postpartum hemorrhage $(\mathrm{PPH})$ is responsible for about $25 \%$ of maternal deaths worldwide. Antifibrinolytic agents, particularly tranexamic acid, have been shown to reduce maternal blood loss and the need for transfusion during delivery in some settings. The aim of this meta-analysis of randomized controlled trials (RCTs) was to evaluate the effectiveness of tranexamic acid for the prevention of PPH after vaginal delivery. The method used as the selection criteria 
was based on RCTs comparing prophylactic use of tranexamic acid after vaginal delivery with controls (either placebo or no treatment). Trials in women undergoing cesarean delivery and trials in women with established postpartum hemorrhage were excluded. The primary outcome was the incidence of primary PPH. The summary step is reported as a summary Relative Risk (RR) with $95 \%$ Confidence Interfal (CI) using the DerSimonian and Laird random-effect model. The results found that four RCTs, including 4671 participants, evaluated the use of $1 \mathrm{~g}$ intravenous (IV) tranexamic acid within 10 minutes of vaginal delivery in addition to oxytocin, umbilical cord traction, and uterine massage, immediately or in the near future for primary PPH prevention, most of which defined as $500 \mathrm{~mL}$ of blood loss in the first 24 hours after delivery, was analyzed. Women who received prophylactic tranexamic acid after vaginal delivery had significantly lower incidence of primary PPH ( 8.7 versus $11.4 \%$; RR $0.61,95 \%$ CI 0.41 0.91 ) and mean differences in blood loss (MD) $84.74 \mathrm{~mL}, 95 \%$ CI 109.76 to 59.72 ). The risk of thrombotic events was not increased in the tranexamic acid group. So the conclusion is that giving prophylactic tranexamic acid $1 \mathrm{~g}$ IV within 10 minutes after vaginal delivery reduces the risk of primary PPH (19).

\section{DISCUSSION}

Postpartum hemorrhage is a complication of the puerperium that is very risky for maternal death, PPH is considered primary if it occurs during the first 24 hours after delivery and secondary if it occurs after 24 hours to 12 weeks after delivery (20). Postpartum hemorrhage is one of the main causes of maternal morbidity and mortality (21). Studies on maternal mortality show that most deaths from PPH are due to delayed and substandard treatment for the diagnosis and management of blood loss. Therefore, reducing the prevalence of severe postpartum hemorrhage remains a challenge that requires early detection of bleeding (22). PPH conditions really need immediate help to minimize the incidence of more serious complications (23). Handling PPH cases requires coordination and cooperation of the health team, including the support of the patient's family with PPH so that case handling can be carried out in a conducive manner (24).

Providing nursing care for patients with postpartum hemorrhage can be done by approaching the values contained in the nursing paradigm. Through the nursing paradigm with its components, namely client / patient, health, nursing and the nurse environment, they can provide comprehensive nursing care for patients with PPH (1). The nursing component elements referred to in the nursing paradigm are a form of health service that is an integral part of professional health services in meeting basic human needs aimed at individuals, families, groups or communities in the range of health and illness. The form of nursing services provided to patients (clients) is in the form of providing nursing care in a situation where the patient is unable, unwilling and does not know in fulfilling basic needs. Nursing includes the attributes, characteristics and actions of nurses who provide nursing care together with patients.

In the case of postpartum hemorrhage patient nursing care, after getting a description of the assessment data, then the nurse enforces the nursing diagnosis, compiles nursing interventions, implements and evaluates the results of the actions that have been taken (15). In connection with 
the nursing intervention stage in this postpartum hemorrhage case, nurses can determine planning, among others in the form of nursing treatment and nursing collaboration.

Postpartum hemorrhage is a case that is very risky to threaten the patient's safety. Through nursing training, nurses can play an independent role to reduce problems that occur to clients (2). Based on the search for the article, actions that can be taken include providing reinforcement of information on the management of postpartum hemorrhage supported by multiple approaches that include the use of an online e-learning platform for maternity emergencies. Postpartum hemorrhage case handling services can be improved by combining existing strategies with the use of an online assessment-based on electronic learning (e-learning) platform. Throughout this online-based learning, evidence from the literature supports the use of a mix of e-learning, instructor-led training, and simulations to effectively educate clinical teams. Early knowledge transfer is effective and potentially more effective through an e-learning format. E_learning learning supports excellence in PPH management by enhancing the continuing education process for maternity care providers as well as nurses. Through the latest knowledge gathered through elearning modules, frontline teams can recognize opportunities to improve protocols, kits and response plans in PPH cases. Before completing the data evaluation, it is assumed that an effective e-learning platform will improve the ability of the care team to manage PPH and therefore, some cases of PPH will be prevented and identified (15)

Another nursing treatment to improve postpartum hemorrhage cases based on the results of this article is foot reflexology which is performed at the 4th stage of delivery. This action is highly recommended because it is safe and has no side effects. In fact, the positive thing that is obtained is that foot reflexiology can control postpartum hemorrhage and post partum pain adaptation (14). Foot reflexiology action like this can have an effect on relaxation, so that stimulated endorphins can increase the production of the hormone oxytocin and allow better uterine contractions.

Based on the nursing paradigm on this nursing component, nursing treatment in postpartum hemorrhage patients can also be done in the form of nursing collaboration. Solving patient health problems in postpartum complications in the form of bleeding events requires the cooperation of a health team which includes doctors, pharmacologists, nurses and others. Collaboration of postpartum hemorrhage management with the medical team can be developed and improved from time to time, to achieve the best solution in the management of PPH cases. Various research findings that have been produced in the treatment of PPH based on article searches include 1) the use of sublingual Misoprostol $800 \mathrm{mcg}$ can change the average $\mathrm{Hb}$ before and after delivery (10); 2) the use of the Digital Postpartum Hemorrhage Management Device (DPHMD) can predict blood loss during the puerperium, so that it can provide fluid therapy accurately (11) ; 3) application of bilateral-contralateral cervical clamps was declared effective and safe as a complementary measure for PPH $(12)$; 4) the use of a suction tube uterine tampon has the effect on stopping bleeding immediately $(13) ; 5)$ the use of bilateral hypogastric artery (BHAL) not only can maintain reproductive function in fertility, but also provide a high chance for patients to survive (16); 6) the use of the Shock Index as a practical and effective method for objective assessment of cases of postpartum hemorrhage (17); 7) found the results of the study that the Carbetocin drug was more effective than oxytocin in reducing intraoperative and postoperative 
bleeding (18) and 8) that giving Tranexamic acid 1 gram IV within 10 minutes of vaginal delivery can reduce the risk of postpartum hemorrhage (19) (25).

The results of the literature search on medical actions in postpartum hemorrhage patients above constitute medical action both as a preventive and curative effort. The role of the nurse related to this medical action is in the form of a collaborative role (nursing collaboration) with the medical team in solving problems for patients who experience bleeding during the puerperium. The implementation of this action certainly has an observation stage to see the success of the action, so to assess the overall medical action from the beginning to the end requires the cooperation of the health team including doctors-nurses as well as other health teams.

The handling of postpartum hemorrhage cases in addition to requiring proper action, also needs to identify cases quickly, this is because the speed of time also determines the patient's prognosis and chances for success. In this regard, it is very important for the family to be directly involved, so that the handling of postpartum hemorrhage cases can be done comprehensively (26)

In accordance with the nursing paradigm, in providing nursing services, nurses use a family centered maternity care (FCMC) philosophical approach. Through this approach, it is very clear that the family is the main support for mothers during the perinatal period, including postpartum mothers in conditions of postpartum complications, namely postpartum hemorrhage. The FCMC philosophy is also a philosophy that underlies an effort to meet the needs of clients as unique individuals and sees each family member as an individual who has special needs and desires that can be met through the nursing process (27). Postpartum hemorrhage mothers will certainly undergo a series of medical management procedures that can provide physical and psychological stimuli for the mother. Therefore, health workers in this case are doctors, including nurses and families, it is very important to provide optimal support, so that patients have adaptive coping both physically and psychologically. Postpartum hemorrhage maternal readiness is of course also a supporting factor for the success of therapy.

\section{CONCLUSION}

Postpartum Hemorrhage is a postpartum complication that requires serious attention, because it has a very high risk of death. The Maternal Mortality Rate (MMR) with one of the main causes is the occurrence of Postpartum Hemorrhage which is still relatively high. Efforts that can be made in the management of postpartum hemorrhage are very complex, in addition to being handled appropriately, they must also be identified quickly, including the process of getting health services. This case requires team work by the health teams, including the medical element (doctor)-nurse, as well as other health teams. The nursing paradigm approach used by nurses in their involvement in providing health services has elements of patient (client), health, environment and nursing. Through nursing elements, the duties and functions of nurses are applied in the form of nursing care using a nursing process approach in which there are nursing intervention steps. Nursing intervention forms in postpartum hemorrhage cases include nursing treatment and nursing collaboration decisions. Based on a literature review from 2019 to 2021, the authors obtained the results of a study which explained that nursing treatment in postpartum hemorrhage patients could be carried out by optimizing the competence of service providers 
(service providers and maternity nurses) in the form of E_Learning, as well as foot reflexiology actions since the 4th stage of delivery. Furthermore, the form of nursing collaboration that can be carried out based on a literature search includes the use of sublingual Misoprostol $800 \mathrm{mcg}$, the Digital Postpartum Hemorrhage Management Device (DPHMD), application of bilateralcontralateral cervical clamps which are declared effective and safe as complementary measures for PPH. The use of a uterine suction tube tampon, bilateral hypogastric artery (BHAL), evaluation of the Shock Index, the effectiveness of the Carbetocin drug rather than oxytocin and administration of the Tranexamic acid 1 gram IV within 10 minutes in vaginal delivery can reduce the risk of postpartum hemorrhage. Hemorrhage can be a stimulus both physically and psychologically, therefore the application of a nursing philosophy based on the philosophy of Family Centered Maternity Care (FCMC) is very important, so that the support of health workers and families enables PPH patients adaptively to undergo the optimal therapy period with the ultimate goal of increasing the success of therapy to prevent maternal death.

\section{REFERENCE}

1. Finlayson, K., Vogel, J.P., Olufemi T. And Oladapo, O.T. What Matters To Women And Healthcare Providers In Relation To Interventions For The Prevention Of Postpartum Haemorrhage: A Qualitative Systematic Review. PLoS One. 2019. https://doi.org/10.1371/journal;

2. Mary, M., Jafarey, S., Dabash, R., Kamal, I., Rabban, A., Abbas, D., Dorocher, J., Tan, Y.L., and Winikoff B. The Safety And Feasibility Of A Family First Aid Approach For The Management Of Postpartum Hemorrhage In Home Births: A Pre-Post Intervention Study In Rural Pakistan. Matern Child Health J. 2021. https://doi.org/10.1007/s10995020-03047-6;

3. Prapawichar, P., Ratinthorn, A., Utriyaprasit, K and Viwatwongkasem. C. Maternal And Health Service Predictors Of Postpartum Hemorrhage Across 14 District, General And Regional Hospitals In Thailand. BMC Pregnancy Childbirth. 2020. https://doi.org/10.1186/s12884-020-2846-x;

4. Ghosh, R., Spindler, H., Morgan, M.C., Cohen, S.R., Begum, N., Gore, A., Mahapatra, T., and Walker DM. Diagnosis And Management Of Postpartum Hemorrhage And Intrapartum Asphyxia In A Quality Improvement Initiative Using Nursementoring And Simulation In Bihar, India. PLoS One. 2019. https://doi.org/10.1371/journal.pone.0216654;

5. Kebede, B.A., Abdo, R.A., Anshebo, A.A., Gebremariam, B.M . No TitlePrevalence And Predictors Of Primary Postpartum Hemorrhage: An Implication For Designing Effective Intervention At Selected Hospitals, Southern Ethiopia. PLoS One. 2019. https://doi.org/10.1371/journal;

6. Hire, M. G., Lange, E. M. S., Vaidyanathan, M., Armour, K. L., \& Toledo P. Effect of Quantification of Blood Loss on Activation of a Postpartum Hemorrhage Protocol and Use of Resources. J Obstet Gynecol Neonatal Nurs. 2020. https://doi.org/10.1016/j.jogn.2020.01.002;

7. Deleu, F., Tharaux, C.D., Dubruille, C.C., Seco, A., Bonnet, M.P. for the E study group. 
A Population-Based Analysis Of French Transfusion Practices For Women Experiencing Severe Postpartum Hemorrhage. Int J Obstet Anesth. 2019. https://doi.org/10.1016/j.ijoa.2019.07.006;

8. Giurazza, F., Angileri, S. A., Corvino, F., Granata, G., Grillo, P., Savoldi, A. P., and Niola R. Postpartum Hemorrhages: Prevention. Semin Ultrasound, CT MRI. 2020. https://doi.org/10.1053/j.sult.2020.09.002;

9. Henriquez., D.D.C.A., Zwart, J.J., Bloemenkamp, K.W.M., Loeff, R.M., Bom, J.G.V.D., Roosmalen, J.J.M.V Zwaginga, J.J. on behalf of the T-1 study G. Fluid Resuscitation During Persistent Postpartum Haemorrhage And Maternal Outcome: Nationwide Cohort Study. Eur J Obstet Gynecol Reprod Biol. 2019. https://doi.org/10.1016/j.ejogrb.2019.01.027;

10. Anger, H.A., Dabash, R., Hassanein, N., Darwish, E., Ramadan, M.C., Medhat Nawar, Charles, D., Breebaart, M., and Winikoff B. A Cluster-Randomized, Non-Inferiority Trial Comparing Use Of Misoprostol For Universal Prophylaxis Vs. Secondary Prevention Of Postpartum Hemorrhage Among Community Level Births In Egypt. BMC Pregnancy Childbirth. 2020. https://doi.org/10.1186/s12884-020-03008-5;

11. Tekela, D.D., Asmare, A.G., Gebremariam, B.M., Assegahegn, C.A., Wami, K.D., Hundessa D. Nemomssa and Simegn GL. Digital Postpartum Hemorrhage Management Device (DPHMD). BMC Pregnancy Childbirth. 2019. https://doi.org/10.1186/s12884019-2601-3;

12. Zhang, Q., Li, T., Xu, Y., and Hu Y. The New Clinical Application Of Bilateral Contralateral Cervix Clamp In Postpartum Hemorrhage: A Retrospective Cohort Study. BMC Pregnancy Childbirth. 2021. https://doi.org/10.1186/s12884-020-03518-2;

13. Hofmeyr, G. J., \& Singata-Madliki M. Novel Suction Tube Uterine Tamponade for treating intractable postpartum haemorrhage: Description of technique and report of 3 cases. BJOG An Int J Obstet Gynaecol. 2020. https://doi.org/10.1111/1471-0528.16169;

14. Sharifi, N., Bahri, N., Talasaz, F. H., Azizi, H., \& Nezami H. The Effect of Foot Reflexology in the Fourth Stage of Labor on Postpartum Hemorrhage and After Pain: A Randomized Controlled Trial Protocol. Adv Integr Med. 2020. https://doi.org/10.1016/j.aimed.2020.06.004;

15. Miner J. Implementing E-Learning to Enhance the Management of Postpartum Hemorrhage. Nurs Women's Heal. 2020. https://doi.org/10.1016/j.nwh.2020.09.010;

16. Içen, M.S., Findik, F.M., Evsen, G.A., Ağaçayak, E., Tunç, S.Y., Evsen, M.S., \& Gül T. Hypogastric Artery Ligation In Postpartum Haemorrhage: A Ten-Year Experience At A Tertiary Care Centre. J Obstet Gynaecol (Lahore). 2020. https://doi.org/10.1080/01443615.2020.1755623;

17. Tanacan, A., Fadiloglu, E., Unal, C., \& Beksac M. Importance Of Shock Index In The Evaluation Of Postpartum Hemorrhage Cases That Necessitate Blood Transfusion. Women Health. 2020. https://doi.org/10.1080/03630242.2020.1802638;

18. Ibrahim, Z.M., Ahmed, W.A.S., El-Hamid, E.M.A., Taha, O.T., \& Elbahie AM. Carbetocin Versus Oxytocin For Prevention Of Postpartum Hemorrhage In Hypertensive Women Undergoing Elective Cesarean Section. Hypertens Pregnancy. 2020. 
https://doi.org/10.1080/10641955.2020.1768268;

19. Saccone, G., Corte, L.D., D’Alessandro,P., Ardino, B., Carbone, L., Raffone, A., Guida, M., Locci, M., Zullo, F., \& Berghella V. Prophylactic Use Of Tranexamic Acid After Vaginal Delivery Reduces The Risk Of Primary Postpartum Hemorrhage. J Matern Neonatal Med. 2019. https://doi.org/10.1080/14767058.2019.1571576;

20. Suarez, S., Conde-Agudelo, A., Borovac-Pinheiro, A., Suarez-Rebling, D., Eckardt, M., Theron, G., \& Burke TF. Uterine Balloon Tamponade for the Treatment of Postpartum Hemorrhage: a Systematic Review and Meta-Analysis. Am J Obstet Gynecol. 2020; Volume 222(Issue 4, April 2020):293.e1-293.e52. https://doi.org/10.1016/j.ajog.2019.11.1287.

21. Jung,Y.M., Kim, H.J., Choi, W.S., Park, J.Y., Seong, N.J., Oh, K.J., Hong, J.S., \& Yoon CJ. CT angiography for the management of postpartum hemorrhage refractory to conservative treatment. J Matern Neonatal Med. 2020. https://doi.org/10.1080/14767058.2020.1846708;

22. Hcini, N., Mchirgui, A., Pomar, L., Beneteau, S., Lambert, T., and Charles G. Early Prediction Of Blood Loss And Postpartum Hemorrhage After Vaginal Delivery By Ultrasound Measurement Of Intrauterine Content. Ultrasound Med Biol. 2020. https://doi.org/10.1016/j.ultrasmedbio.2020.07.017;

23. Raimann, F.J., Möllman, C.J., Jennewein, L., Sonntagbauer, M., Raddatz, L.M., Louwen, F., Zacharowski, K., Weber CF. Influence Of The WOMAN Trial On National Wide Standard Operating Procedures For Treatment Of Postpartum Hemorrhage. J Gynecol Obstet Hum Reprod. 2019. http://dx.doi.org/10.1016/j.jogoh.2019.01.010;

24. Zea-Prado, F., Hernández-Pacheco, J., Ortiz-Ramírez, M., Gutiérrez-Marín, A., EstradaGutierrez, G., Escobar, M., Seligson-Rios, A., \& Espino-y-Sosa S. Initial management of primary postpartum hemorrhage: a survey. J Matern Neonatal Med. 2019. https://doi.org/10.1080/14767058.2019.1671342;

25. Osoti, A. O., Vogel, J. P., Oladapo, O. T., Qureshi, Z. P., \& Gülmezoglu AM. Tranexamic acid for treatment of postpartum haemorrhage. Obstet Gynaecol Reprod Med. 2019; Volume 29(Issue 5):146-7. https://doi.org/10.1016/j.ogrm.2019.02.004.

26. Ristanti, A.D., dan Masita ED. Efektivitas Psikoedukasi Dan Family Centered Maternity Care. Pada Ibu Postpartum. J Kesehat Poltekkes Palembang. 2020;15. https://doi.org/10.36086/jpp.v15i1.553.

27. Mayasari, S.I., dan Nicky Danur Jayanti ND. Penerapan Edukasi Family Centered Maternity Care (FCMC) terhadap Keluhan Ibu Postpartum Melalui Asuhan Home Care. JNK J Ners Dan Kebidanan. 2019. https://doi.org/10.26699/jnk.v6i2.ART.p135-141; 